\title{
Theoretical constraints on the cross-tail width of bursty bulk flows
}

\section{X. Chen}

CAS Key Laboratory of Geospace Environment, Department of Geophysics and Planetary Sciences, School of Earth and Space Sciences, University of Science and Technology of China, Hefei, Anhui 230026, China

Correspondence to: C. X. Chen (chuxin@ustc.edu.cn)

Received: 22 July 2013 - Revised: 12 November 2013 - Accepted: 12 November 2013 - Published: 11 December 2013

\begin{abstract}
The characteristic cross-tail width of bursty bulk flows (BBFs) in earth's plasma sheet was investigated at two stages of its life, one at its onset, the other when it is fully developed. Equilibrium domains with gradient of magnetic field are constructed. Interchange instability analysis of such domains yields the most unstable mode with the half wave length comparable with the observed cross-tail width of a flow burst and the inverse of growth rate comparable with its duration. The thickness of the plasma sheet for the most unstable mode is also comparable to the width of BBFs in the north-south direction. We found that viscosity, the dimension of the unstable domain, the thickness of the plasma sheet and gradient of the magnetic field together determine the most unstable mode. The ion Larmor radius plays an important role in viscosity as half effective mean free path. For a fully developed flow, however, velocity-caused pressure difference between the leading and trailing sides of a flow burst also plays a role. The equatorial cross section of flow is reshaped and its cross-tail width is changed as well. Representing the surrounding medium with empirical magnetic field and plasma models, the force balance of the fast flow is analyzed. The cross-section area of flow burst is estimated to be one to several square earth radii, and the cross-tail width of fast flow is estimated to be 1 to 3 earth radii, which is consistent with observations of BBFs.
\end{abstract}

Keywords. Magnetospheric physics (plasma sheet)

\section{Introduction}

Any given region of the earth's plasma sheet is nearly stagnant most of the time but experiences short intervals of high-speed flows (Baumjohann et al., 1990; Angelopoulos et al., 1992). These high-speed flows organize themselves into 10 min timescale flow enhancements which are termed bursty bulk flow (BBF) events. Inside the BBFs there exist several flow velocity peaks of very large amplitude (above $400 \mathrm{~km} \mathrm{~s}^{-1}$ ) with a characteristic timescale on the order of a minute; these events are called flow bursts (FBs). A FB is usually accompanied by the dipolarization of magnetic field and the heating of the plasma. Although they highly correlate with substorms, BBFs are observed to occur at all levels of geomagnetic activity.

Another widely observed and intensely investigated feature in the magnetotail, dipolarization front (DF), is closely associated with flow burst (Runov et al., 2009; Sergeev et al., 2009; Ohtani et al., 2004; Eastwood et al., 2005; Wolf et al., 2009; Zhou et al., 2009; Ashour-Abdalla et al., 2010; Sitnov and Swisdak, 2011). DFs are characterized by a strong and steep increase of the magnetic field component normal to the neutral plane. They usually appear on the leading sides of flow bursts. With the ion inertia length dimension, DFs have low density and high speed.

To interpret the high-speed flows in the plasma sheet, Chen and Wolf (1993) proposed a "bubble" model (i.e., bubbles are plasma-depleted magnetic flux tubes). Under the influence of the interchange instability, these bubbles accelerate earthward, producing BBFs. The bubble picture naturally explains most of the characteristics of BBFs and has been supported later by several ground-based and ionospheric observations (e.g., Sergeev et al., 1999, 2000; Kauristie et al., 2000).

Observations show that the earthward transport of magnetic flux, mass and energy are mainly accomplished by BBFs (60-100 \%) (Angelopoulos et al., 1994). The crosstail widths of BBFs were found to be 1 to 3 earth radii $\left(R_{\mathrm{E}}\right)$ (Angelopoulos et al., 1996; Sergeev et al., 1996; Nakamura et al., 2004). Theoretically the cross-tail width of bubble was originally estimated as on the order of $1 R_{\mathrm{E}}$ (Pontius and Wolf, 1990; Chen and Wolf, 1999) based on the argument 
that smaller bubble would diffuse quickly by drifting particles before reaching the inner magnetosphere.

Recently, theoretical study of the cross-tail width of fast flow is very active. A 3-D magnetohydrodynamics (MHD) simulation has tried different widths of bubble (Birn et al., 2004). Their study has shown that the speed of flow is inversely correlated with the width of bubble and that the equatorial cross section of bubble has mushroom shape. Global MHD simulations have produced fast flows with the crosstail width of a few $R_{\mathrm{E}}$ (Wiltberger et al., 2000; Raeder et al., 2010; Ge et al., 2011). A 2-D MHD simulation by Guzdar et al. (2010) also produced the mushroom shape cross section of bubble. They treated the cross-tail width by the seed interchange perturbation scale. A 3-D MHD simulation by Lapenta and Bettarini (2011) suggested that the BBF crosstail width is determined by other instabilities, different from the interchange instability. On the other hand, kinetic simulations have also produced bubbles with widths of several tens of the ion inertia lengths (Nakamura et al., 2002; Pritchett and Coroniti, 1997, 2010, 2011). These results are marginally consistent with the MHD estimates for the magnetotail. The most recent 3-D PIC simulation results by Pritchett and Coroniti (2013) suggested that the increase of the characteristic cross-tail width of BBFs may be a nonlinear effect.

What exactly constrains the width of fast flow is still an unsolved problem. In present study, we will try to pin point the factors that could affect the characteristic cross-tail width of BBFs. We would like to investigate the problem using MHD but with a viscosity that is extracted from the microscopic property of plasma particle. Two stages of bubble life would be studied, onset and full development. In Sect. 2 an instability analysis will be demonstrated to indicate the most unstable mode of interchange. In Sect. 3 an estimation of the cross-tail width of a fully developed flow burst will be presented. And finally discussion and summary will be given in Sect. 4.

\section{Mode of interchange instability}

We first construct an unstable domain. Defining Cartesian coordinates with the $x$ axis pointed tailward, the $y$ axis pointed from dusk to dawn. Symmetry about equatorial plane is assumed in this domain. To get an analytical solution we would further assume the medium is incompressible, bearing in mind that an incompressible fluid gives at least as much stability as a compressible one in the same configuration (George, 1979).

Assuming the medium is perfectly conductive, the Faraday's law gives

$$
\frac{\partial \boldsymbol{B}}{\partial t}=\nabla \times(\boldsymbol{u} \times \boldsymbol{B})
$$

where $\boldsymbol{B}$ is magnetic field and $\boldsymbol{u}$ is velocity. Imposing the incompressible condition, Eq. (1) becomes

$$
\frac{\partial \boldsymbol{B}}{\partial t}=(\boldsymbol{B} \cdot \nabla) \boldsymbol{u}-(\boldsymbol{u} \cdot \nabla) \boldsymbol{B} .
$$

Let the perturbation of magnetic field be $\delta \boldsymbol{B}$, then Eq. (2) stands as

$$
\frac{\partial \delta \boldsymbol{B}}{\partial t}=\left(\boldsymbol{B}_{0} \cdot \nabla\right) \boldsymbol{u}-(\boldsymbol{u} \cdot \nabla) \boldsymbol{B}_{0},
$$

where subscript 0 represents the background unstable medium and only first order terms are kept.

We would like to simplify the problem to a twodimensional one. Averaging over the thickness of the plasma sheet in the $z$ direction, Eq. (3) then reduces to

$\frac{\partial \delta B}{\partial t}=-u_{x} \frac{\mathrm{d} B_{0}}{\mathrm{~d} x}$,

where $u_{x}$ is the $x$ component of velocity, $B_{0}$ stands for $B_{z 0}$; we have dropped the subscript $z$ for simplicity. The $z$ component of velocity and the $x$ and $y$ components of the magnetic field are cancelled out because of the symmetry about the equatorial plane.

We further assume the background medium is in equilibrium state, that is

$-\nabla\left(p_{0}+\frac{\boldsymbol{B}_{0}^{2}}{2 \mu_{0}}\right)+\frac{\left(\boldsymbol{B}_{0} \cdot \nabla\right) \boldsymbol{B}_{0}}{\mu_{0}}=0$,

where $p_{0}$ is thermal pressure and $\mu_{0}$ is magnetic permeability. Averaging the above equation over the thickness of the plasma sheet in the $z$ direction, we get

$$
\begin{aligned}
& -\frac{\partial}{\partial x}\left(p_{0}+\frac{B_{0}^{2}}{2 \mu_{0}}\right)-\frac{B_{0}^{2}}{\mu_{0} R}=0, \\
& -\frac{\partial}{\partial y}\left(p_{0}+\frac{B_{0}^{2}}{2 \mu_{0}}\right)=0,
\end{aligned}
$$

where $R$ is an averaged curvature radius of the magnetic field, it is a function of $x$ in the domain $(-d \leq x \leq d)$ of interest.

The $x$ component of momentum equation is

$$
\begin{aligned}
\rho \frac{\partial u_{x}}{\partial t}+\rho(\boldsymbol{u} \cdot \nabla) u_{x} & =-\frac{\partial}{\partial x}\left(p+\frac{\boldsymbol{B}^{2}}{2 \mu_{0}}\right)+\frac{(\boldsymbol{B} \cdot \nabla) B_{x}}{\mu_{0}} \\
& +\mu \nabla^{2} u_{x}+\left(\frac{\partial u_{x}}{\partial x}+\frac{\partial u_{x}}{\partial x}\right) \frac{\mathrm{d} \mu}{\mathrm{d} x}
\end{aligned}
$$

and the $y$ component of momentum equation is

$$
\begin{aligned}
\rho \frac{\partial u_{y}}{\partial t} & +\rho(\boldsymbol{u} \cdot \nabla) u_{y}=-\frac{\partial}{\partial y}\left(p+\frac{\boldsymbol{B}^{2}}{2 \mu_{0}}\right)+\frac{(\boldsymbol{B} \cdot \nabla) B_{y}}{\mu_{0}} \\
& +\mu \nabla^{2} u_{y}+\left(\frac{\partial u_{y}}{\partial x}+\frac{\partial u_{x}}{\partial y}\right) \frac{\mathrm{d} \mu}{\mathrm{d} x}
\end{aligned}
$$


where $p$ is thermal pressure, $\rho$ is density, $\mu$ is viscosity, $u_{y}$ is the $y$ component of velocity, $B_{x}$ and $B_{y}$ are the $x$ and $y$ components of the magnetic field respectively.

Averaging Eqs. (8) and (9) over the thickness of the plasma sheet in the $z$ direction, substituting Eqs. (6) and (7) into Eqs. (8) and (9) and keeping first order terms, we have then, the $x$ component of momentum equation for perturbation as

$$
\begin{aligned}
\rho \frac{\partial u_{x}}{\partial t} & =-\frac{\partial}{\partial x}\left(\delta p+\frac{2 B_{0} \delta B}{2 \mu_{0}}\right)-\frac{2 B_{0} \delta B}{\mu_{0} R}+\mu \nabla^{2} u_{x} \\
& +\left(\frac{\partial u_{x}}{\partial x}+\frac{\partial u_{x}}{\partial x}\right) \frac{\mathrm{d} \mu}{\mathrm{d} x},
\end{aligned}
$$

and the $y$ component of momentum equation for perturbation as

$$
\begin{aligned}
\rho \frac{\partial u_{y}}{\partial t} & =-\frac{\partial}{\partial y}\left(\delta p+\frac{2 B_{0} \delta B}{2 \mu_{0}}\right)+\mu \nabla^{2} u_{y} \\
& +\left(\frac{\partial u_{y}}{\partial x}+\frac{\partial u_{x}}{\partial y}\right) \frac{\mathrm{d} \mu}{\mathrm{d} x},
\end{aligned}
$$

where $\delta p$ is the perturbation of thermal pressure.

We seek solutions whose dependence on $y$ and $t$ are given by

$\exp (i k y+n t)$

where $k$ is wave number and $n$ is growth rate (Chandrasekhar, 1961). Then Eq. (4) stands as

$n \delta B=-u_{x} D B_{0}$,

where $D$ represents the derivative with respect to $x$. And Eqs. (10), (11) become

$$
\begin{aligned}
\rho n u_{x} & =-D \delta p-\frac{\delta B}{\mu_{0}} D B_{0}-\frac{B_{0}}{\mu_{0}} D \delta B-\frac{2 B_{0} \delta B}{\mu_{0} R} \\
& +\mu\left(D^{2}-k^{2}\right) u_{x}+2(D \mu)\left(D u_{x}\right), \\
\rho n u_{y} & =-i k \delta p-i k \frac{B_{0} \delta B}{\mu_{0}}+\mu\left(D^{2}-k^{2}\right) u_{y} \\
& +\left(D u_{y}+i k u_{x}\right)(D \mu) .
\end{aligned}
$$

Multiplying Eq. (15) by $i k$, we have

$$
\begin{aligned}
i k \rho n u_{y} & =k^{2} \delta p+k^{2} \frac{B_{0} \delta B}{\mu_{0}}+\mu\left(D^{2}-k^{2}\right) i k u_{y} \\
& +\left[D\left(i k u_{y}\right)-k^{2} u_{x}\right](D \mu) .
\end{aligned}
$$

The incompressible condition can be represented by

$\nabla \cdot \boldsymbol{u}=\frac{\partial u_{x}}{\partial x}+\frac{\partial u_{y}}{\partial y}=D u_{x}+i k u_{y}=0$,

where averaging over the thickness of the plasma sheet in the $z$ direction has been taken.
Substituting Eq. (17) into Eq. (16), one gets

$$
\begin{aligned}
k^{2} \delta p & =-\rho n D u_{x}+\mu\left(D^{2}-k^{2}\right) D u_{x} \\
& +(D \mu)\left(D^{2}+k^{2}\right) u_{x}-k^{2} \frac{B_{0} \delta B}{\mu_{0}} .
\end{aligned}
$$

We would like to impose no-slip condition on boundary $\left(u_{y}=0\right.$, at $\left.x= \pm d\right)$. From Eq. (17), this boundary condition stands as

$u_{x}=D u_{x}=0(x= \pm d)$.

Equation (A13) of the Appendix gives the equation satisfied by $n$

$I_{1} n^{2}+I_{3} n-I_{2}=0$,

where $I_{1}, I_{2}$ and $I_{3}$ are defined by Eqs. (A14), (A15) and (A16), respectively.

The $n$ value for the most unstable mode is given by

$n=\frac{-I_{3}+\sqrt{I_{3}^{2}+4 I_{1} I_{2}}}{2 I_{1}}$.

Based on the variational principle in the Appendix, this problem can be tackled by variation method. In applying the Ritz method to our problem, we take $u_{x}$ as

$u_{x}=(a+b x)\left(1-\frac{x^{2}}{d^{2}}\right)^{2} \exp (i k y+n t)$,

which satisfies the boundary condition (Eq. 19). Since $a$ will appear in both numerator and denominator of Eq. (21), we would like to set $a=1$. The problem now is to find out the maximum value of $n$ for a given background medium.

Following the treatment of Chen and Wolf (1999), we would take viscosity as

$\mu=\frac{1}{3}(\rho \bar{u} \bar{\lambda})$,

where $\bar{u}$ is the mean thermal speed and $\bar{\lambda}$ is the mean free path length which in the collisionless earth's magnetosphere can be taken as

$\bar{\lambda}=2 a_{c}$,

where $a_{c}$ is ion gyroradius.

It is worthwhile to give a validity justification of a viscosity as expressed by Eq. (23). The mean thermal speed $\bar{u}$ is expressed as

$\bar{u}=\left(\frac{8 K T_{0}}{\pi m}\right)^{1 / 2}$,

where $K$ is Boltzmann's constant, $T_{0}$ is the temperature of background medium and $m$ is the mass of proton. The ion gyroradius $a_{c}$ is expressed as

$a_{c}=\frac{m \bar{u}}{e B_{0}}$, 
where $e$ is the electric charge of an electron. From Eq. (23), kinetic viscosity is thus

$v=\frac{1}{3}(\bar{u} \bar{\lambda})$.

The typical plasma sheet parameters at $20 R_{\mathrm{E}}$ down tail are given in Table 1 of the work by Borovsky et al. (1997). Taking ion temperature as $5 \mathrm{keV}$ from the third row and magnetic field strength as $10^{-4}$ gauss from the fifth row of the table, substituting these two values into Eqs. (25) and (26) for mean thermal speed and ion gyroradius, the kinetic viscosity from Eq. (27) would be $8.06 \times 10^{11}$ square meter per second. This matches quite well with the eddy viscosity value of $8 \times 10^{15}$ square centimeter per second presented in the last row of the same table.

Following the works of Schindler (1972), Pritchett and Coroniti (2010), we construct the unstable equilibrium background medium domain by specifying the vector potential as

$A_{0 y}(x, z)=B_{0 l} L \ln \{\cosh [F(X)(z / L)] / F(X)\}$,

where $L$ is an adjustable parameter which when combined with $F(X)$ as $L / F(X)$ represents the half thickness of the plasma sheet, $B_{0 l}$ is the magnetic field strength of the lobe. The function $F(X)$ is given as

$F(X)=1.0-(x+d) \mathrm{SP}$,

where SP is another adjustable parameter which controls the gradient of the background magnetic field. In the domain of interest, $D B_{0}^{2}$ is positive in the tailward direction when SP is positively defined. The corresponding number density is given by

$N(x, z)=N_{0} F^{2}(X) \cosh ^{-2}[F(X)(z / L)]$.

We are interested in the interchange instability taking place down tail from earth. To understand it, we set the value of parameters of the background medium as those observed in the plasma sheet. The temperature is set to $5 \mathrm{keV}$ and is fixed inside the domain of interest, density $N_{0}$ is set to 0.2 per cubic centimeter. The magnetic field strength of the lobe $B_{0 l}$ is set to $20 \mathrm{nT}$ for self-consistence. The calculations are carried out in a two-dimensional parameter space specified by $L$ and SP. An example domain is shown in Fig. 1.

To investigate the effect of the gradient of the magnetic field, SP is set to $0.15,0.2$ and 0.25 . For different SP, the equatorial magnetic field strengths are displayed in Fig. 2. The $x$ dimension of an equilibrium domain is determined by setting the density on the $x=+d$ boundary one order of magnitude smaller than that on the $x=-d$ boundary as indicated in Fig. 3. This is because the density at $x=+d$ boundary represents the density of the lobe if we consider magnetic reconnection as the cause to an unstable domain. The larger the SP is, the smaller the $x$ dimension of the domain $d$ is.

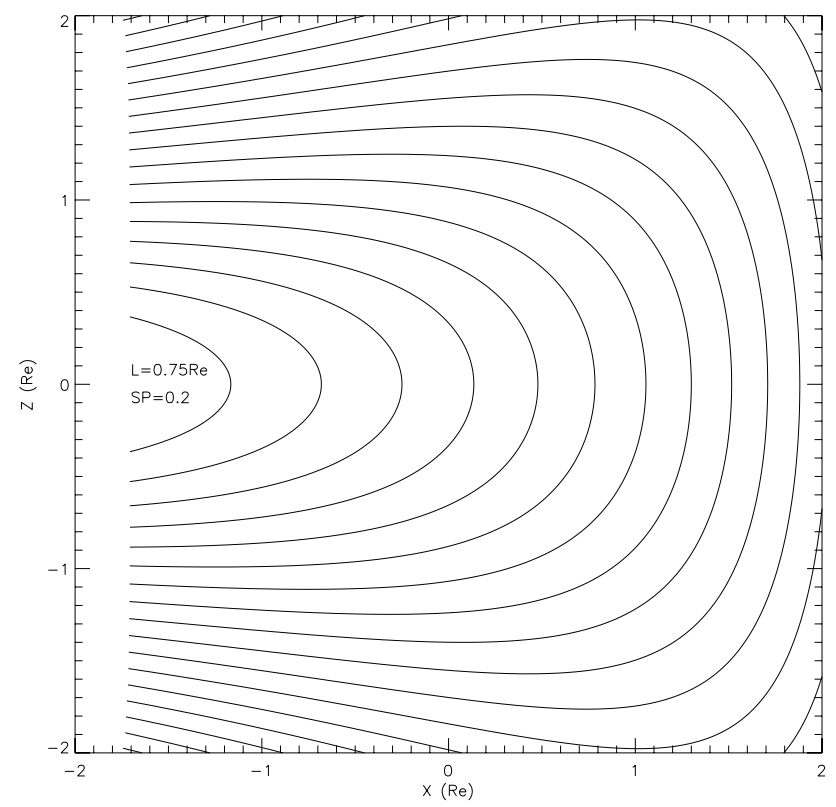

Fig. 1. An example unstable domain $\left(L=0.75 R_{\mathrm{E}}, \mathrm{SP}=0.2\right)$.

In a given background medium domain (one labeled line in Figs. 2 and 3 as specified by $L$ and SP), we can find out the maximum value of $n$ for a given $k$ by choosing the right $b$ value in Eq. (22). Figure 4 shows such plots of different background medium domains for the $L=0.75 R_{\mathrm{E}}$ case. Each line (corresponding to one background medium domain) has its maximum $n$ value at certain $k$. The larger the dimension of domain $d$ is, the smaller the most unstable wave number $k$ is, as well as the smaller the growth rate of the most unstable mode $n$ is. The most unstable wave number $k$ has special meaning, since the half wave length $\pi / k$ gives the width of the most fast growing earthward motion in cross-tail direction. Line ( $\mathrm{SP}=0.15, L=0.75 R_{\mathrm{E}}$ ) has the maximum $n$ as $0.0129 / \mathrm{s}$ and the half wave length as $2.94 R_{\mathrm{E}}\left(k=1.07 / R_{\mathrm{E}}\right)$. Line ( $\mathrm{SP}=0.2, L=0.75 R_{\mathrm{E}}$ ) has the maximum $n$ as $0.0172 / \mathrm{s}$ and the half wave length as $2.2 R_{\mathrm{E}}\left(k=1.43 / R_{\mathrm{E}}\right)$. Line ( $\mathrm{SP}=0.25, L=0.75 R_{\mathrm{E}}$ ) has the maximum $n$ as $0.0215 / \mathrm{s}$ and the half wave length as $1.76 R_{\mathrm{E}}\left(k=1.79 / R_{\mathrm{E}}\right)$. Three dashed lines show the positions for the half wave length as $1 R_{\mathrm{E}}$, $2 R_{\mathrm{E}}$ and $3 R_{\mathrm{E}}$. Figure 4 may also be interpreted as that the reduction of the magnetic field gradient reduces the growth rate and the unstable wave number.

To investigate the effect of the thickness of the plasma sheet, a similar plot ( $n$ vs. $k$ ) of different background medium domains for the $\mathrm{SP}=0.2$ case is shown in Fig. 5. Line ( $L=1.0 R_{\mathrm{E}}, \mathrm{SP}=0.2$ ) has the maximum $n$ as $0.0217 / \mathrm{s}$ and the half wave length as $2.15 R_{\mathrm{E}}\left(k=1.46 / R_{\mathrm{E}}\right)$. Line $(L=$ $0.5 R_{\mathrm{E}}, \mathrm{SP}=0.2$ ) has the maximum $n$ as $0.012 / \mathrm{s}$ and the half wave length as $2.23 R_{\mathrm{E}}\left(k=1.41 / R_{\mathrm{E}}\right)$. Line $\left(L=0.4 R_{\mathrm{E}}\right.$, $\mathrm{SP}=0.2$ ) has the maximum $n$ as $0.00972 / \mathrm{s}$ and the half wave length as $2.24 R_{\mathrm{E}}\left(k=1.40 / R_{\mathrm{E}}\right)$. Line $\left(L=0.75 R_{\mathrm{E}}\right.$, $\mathrm{SP}=0.2$ ) is also plotted for comparison. The conclusion is 


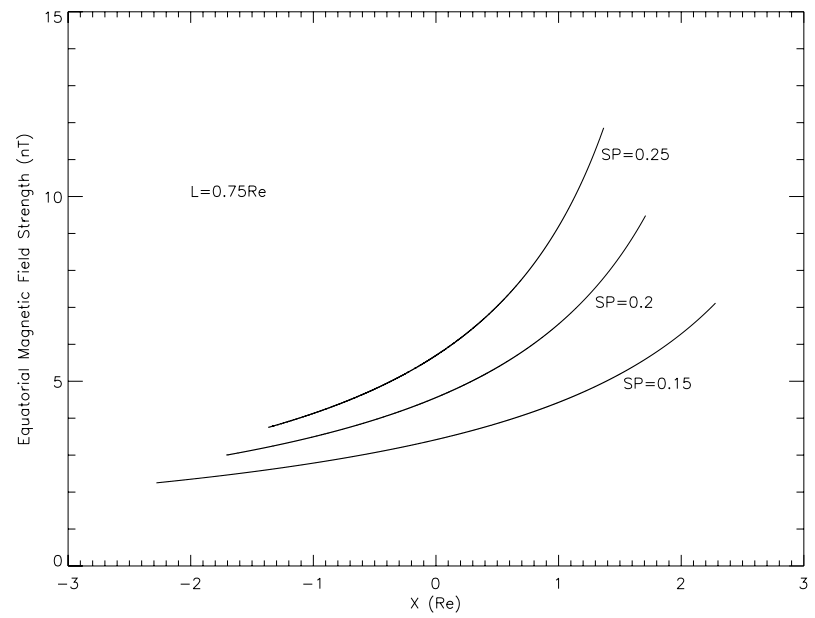

Fig. 2. Background magnetic field magnitude in the domain $(L=$ $0.75 R_{\mathrm{E}}$ ). Each labeled line indicates an unstable domain. Ends of each line indicate the boundaries of the domain.

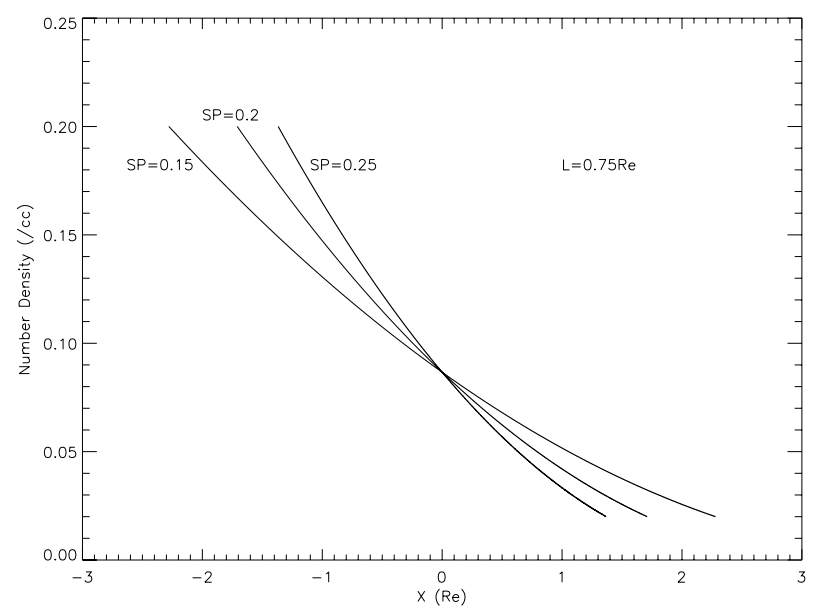

Fig. 3. Background number density in the domain $\left(L=0.75 R_{\mathrm{E}}\right)$. Each labeled line indicates an unstable domain. Ends of each line indicate the boundaries of the domain.

that their half wave lengths are almost the same, but the maximum of growth rate reduces with the reduction of the thickness of the plasma sheet. It is interesting to find out that the thickness ( 0.8 to $\left.2 R_{\mathrm{E}}\right)$ of the plasma sheet $(2 L)$ is comparable to the width (1.5 to $2 R_{\mathrm{E}}$ ) of BBFs in the north-south direction (Nakamura et al., 2004).

\section{Width of bursty bulk flows}

We now turn to the fully developed flows. Applying the momentum equation to the flow in the plasma sheet, we have

$$
\begin{aligned}
\rho \frac{\partial}{\partial t} \boldsymbol{u}+\rho(\boldsymbol{u} \cdot \nabla) \boldsymbol{u} & =-\nabla\left(p+\frac{B^{2}}{2 \mu_{0}}\right)+\frac{1}{\mu_{0}}(\boldsymbol{B} \cdot \nabla) \boldsymbol{B} \\
& +\mu \nabla^{2} \boldsymbol{u} .
\end{aligned}
$$

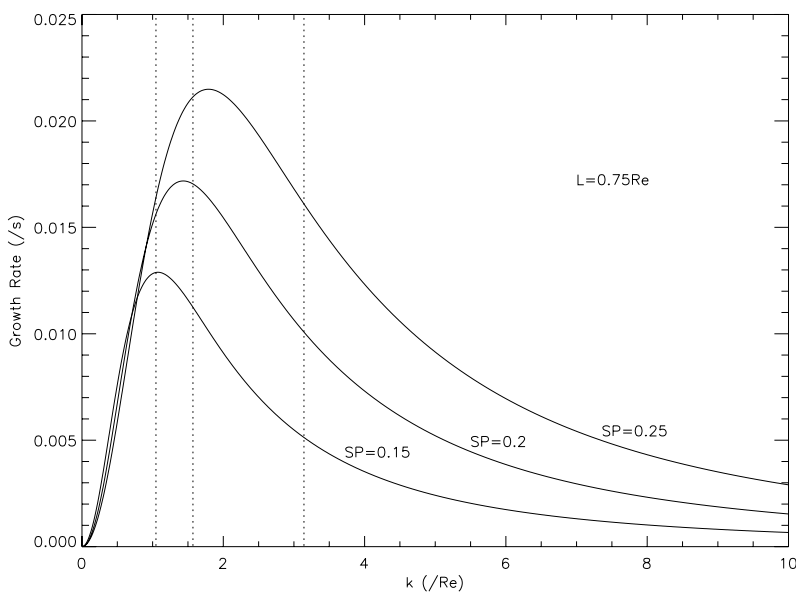

Fig. 4. Growth rate as the function of wave number for each background medium $\left(L=0.75 R_{\mathrm{E}}\right)$. Each labeled line indicates an unstable domain.

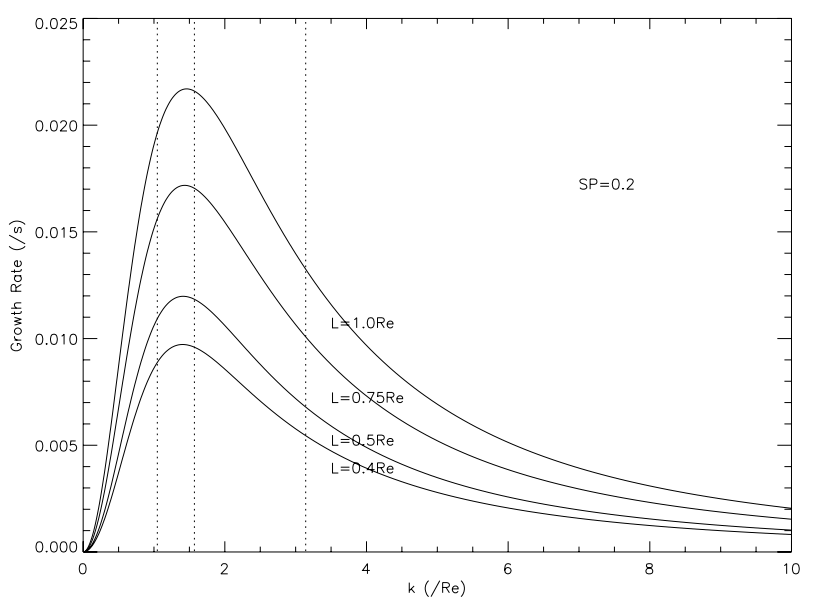

Fig. 5. Growth rate as the function of wave number for each background medium $(\mathrm{SP}=0.2)$. Each labeled line indicates an unstable domain.

Because of the high beta property of the plasma sheet, flow pattern in bursty bulk flows channel is complicated. Individual flow burst would produce turbulence in its wake (Borovsky et al., 1997; Antonova et al., 1999). Except for the first flow burst in BBFs, each following flow burst moves in the wake of its preceding one. Instead of solving this problem directly, we would deal with two extreme cases, with the understanding that the real situation in BBFs is somewhere between these two extremes. One case is that only one isolated flow burst moves in a somewhat rest background medium; the other case is that flow bursts chase each other so closely that BBFs can be regarded as a uniform flow channel in the flow direction and all physical quantities can change only in the across channel direction. 
An individual flow burst would experience three periods in its life (i.e., speed up, cruise and break). In its cruise period, the flow is fully developed, the speed of flow reaches its maximum $\left(\geq 400 \mathrm{~km} \mathrm{~s}^{-1}\right)$. The cruise phase is expected to last most of its life. In the speed up period, magnetic tension can not be balanced by the gradients of plasma pressure and magnetic pressure, a bubble experiences acceleration. On its way towards earth, the field line gets dipolarized, and magnetic tension reduces. Consequently a rough force balance among magnetic tension, gradient of total pressure and drag force is established. This balance would be maintained by the adjustment of dipolarization of magnetic field line. As the bubble approaches the region of much higher gradient of total pressure near earth, the rough force balance can not be maintained because the reversal process of dipolarization to obtain more magnetic tension is not possible when the flow has earthward velocity, the bubble would break. The recent observation of the oscillation of flow at its equilibrium position (Panov et al., 2010) could be viewed as the indirect evidence of long lasting cruise phase. We would like to think that most of the observed BBFs are the cruise flows or fully developed flows.

In the reference frame of flow burst, the flow is in a steady state in this period. Thus Eq. (31) reduces to

$\rho(\boldsymbol{u} \cdot \nabla) \boldsymbol{u}=-\nabla\left(p+\frac{B^{2}}{2 \mu_{0}}\right)+\frac{1}{\mu_{0}}(\boldsymbol{B} \cdot \nabla) \boldsymbol{B}+\mu \nabla^{2} \boldsymbol{u}$

We further assume that the background medium is in equilibrium as expressed by Eq. (5).

The force balance between the flow and the background medium in the cross-tail direction stands as

$p+\frac{B^{2}}{2 \mu_{0}}=p_{0}+\frac{B_{0}^{2}}{2 \mu_{0}}$.

\subsection{Equatorial size of an isolated flow burst}

For an isolated flow burst, Eq. (32) states that magnetic tension balances the negative of total pressure gradient, negative of velocity-caused pressure gradient and viscosity.

Substituting Eqs. (5) and (33) into Eq. (32), one gets

$\frac{1}{\mu_{0}}(\boldsymbol{B} \cdot \nabla) \delta \boldsymbol{B}+\frac{1}{\mu_{0}}(\delta \boldsymbol{B} \cdot \nabla) \boldsymbol{B}_{0}-\rho(\boldsymbol{u} \cdot \nabla) \boldsymbol{u}+\mu \nabla^{2} \boldsymbol{u}=0$,

where $\delta \boldsymbol{B}$ is the magnetic field difference between the flow and the background medium (this variable has the same meaning as in Sect. 2 but is much larger now). The flow induced drag force $\boldsymbol{F}_{\mathrm{d}}$ is defined as

$\boldsymbol{F}_{\mathrm{d}}=-\rho(\boldsymbol{u} \cdot \nabla) \boldsymbol{u}+\mu \nabla^{2} \boldsymbol{u}$

Equation (34) indicates that magnetic buoyancy force balances the flow induced drag force which consists of velocity induced pressure difference between the front and rear sides of a FB and viscosity at its flanks. It is this equation that will be used to estimate the equatorial size of an isolated flow burst.

In the reference frame moving with the center of a flow burst, we define Cartesian coordinates with the $x$ axis pointed earthward, the $y$ axis pointed from dawn to dusk, and the origin located at the center of the flow burst. Then the velocity is assumed to be a Gaussian distribution as

$\boldsymbol{u}=\left[u(x) \exp \left(-\frac{y^{2}}{2 \sigma^{2}}\right)-U\right] \boldsymbol{e}_{x}$,

where $U$ is the amplitude of the flow. Conventionally, we would like to define the cross-tail width of flow $\Delta Y$ as the width between half maximum value points of the Gaussian distribution, that is,

$\frac{\Delta Y}{2}=1.1774 \sigma$.

Further, we assume that a flow burst has its $x$ dimension as $\Delta X$, that is,

$\left(\frac{\partial u}{\partial x}\right)_{x=0, y=0}=\left[\frac{\partial u(x)}{\partial x}\right]_{x=0} \approx-\frac{U}{\Delta X}$.

Substituting Eqs. (36), (37) and (38) into Eq. (35), we have

$\boldsymbol{F}_{\mathrm{d}}=\left[-\frac{\rho_{0} U^{2}}{2 \Delta X}-\mu \frac{2(1.1774)^{2} U}{(\Delta Y)^{2}}\right] \boldsymbol{e}_{x}$.

Let the equatorial cross-section area of a $\mathrm{FB}$ as $\Delta S$, one has

$\Delta S=\Delta X \Delta Y$

Let the ratio of $\Delta X$ to $\Delta Y$ as $r$, then we have

$r=\frac{\Delta X}{\Delta Y}$

Substituting Eqs. (40) and (41) into Eq. (39), we obtain

$$
\boldsymbol{F}_{\mathrm{d}}=-\frac{\rho_{0} U}{(\Delta S)^{1 / 2}}\left[\frac{U}{2(r)^{1 / 2}}+\frac{2 \mu(1.1774)^{2} r}{\rho_{0}(\Delta S)^{1 / 2}}\right] \boldsymbol{e}_{x}
$$

For a fixed equatorial cross-section area $\Delta S$, the ratio $r$ would take such a value so that the flow induced drag force $\boldsymbol{F}_{\mathrm{d}}$ reaches its extreme value, probably minimum. This condition leads to

$r=\left[\frac{\rho_{0} U(\Delta S)^{1 / 2}}{8 \mu(1.1774)^{2}}\right]^{2 / 3}$.

where $\mu$ is taken from Eq. (23).

Substituting Eqs. (23)-(26) back into Eqs. (43) and (42), we get

$r=\left[\frac{3 \pi e B_{0} U(\Delta S)^{1 / 2}}{128 K T_{0}(1.1774)^{2}}\right]^{2 / 3}$, 


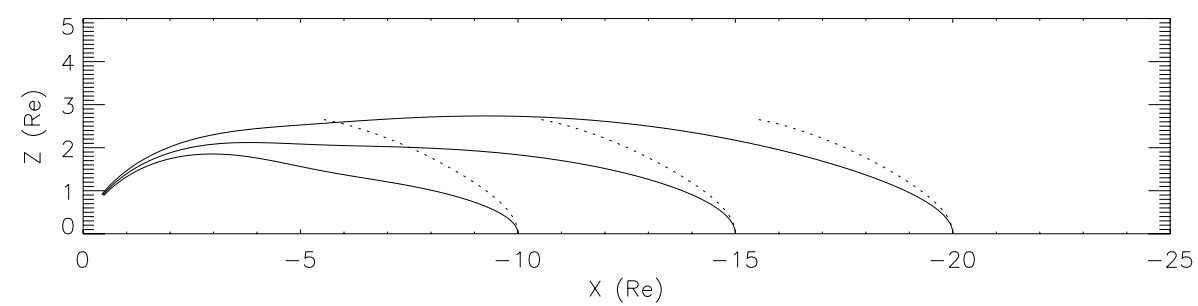

Fig. 6. Comparison between the special field line (dashed lines) taken from Tsyganenko (96) magnetospheric magnetic field model (with the parameters set as the solar wind density 6 per cubic centimeter, velocity $380 \mathrm{~km} \mathrm{~s}^{-1}$, IMF $B_{z}=5 \mathrm{nT}$ and Dst $=0 \mathrm{nT}$ ) with equatorial plane crossing point at $-10 R_{\mathrm{E}}$ and the field lines taken from the same model (with the parameters set as the solar wind density 6 per cubic centimeter, velocity $380 \mathrm{~km} \mathrm{~s}^{-1}$, IMF $B_{z}=-5 \mathrm{nT}$ and Dst $=-80 \mathrm{nT}$ ) with equatorial plane crossing point at $-10 R_{\mathrm{E}},-15 R_{\mathrm{E}}$ and $-20 R_{\mathrm{E}}$.

and

$\boldsymbol{F}_{\mathrm{d}}=-\frac{3 \rho_{0} U^{5 / 3}\left(128 K T_{0}\right)^{1 / 3}(1.1774)^{2 / 3}}{4\left(3 \pi e B_{0}\right)^{1 / 3}(\Delta S)^{2 / 3}} \boldsymbol{e}_{x}$.

Substituting Eq. (45) into Eq. (34), we obtain

$$
\begin{aligned}
& \frac{1}{\mu_{0}}(\boldsymbol{B} \cdot \nabla) \delta \boldsymbol{B}+\frac{1}{\mu_{0}}(\delta \boldsymbol{B} \cdot \nabla) \boldsymbol{B}_{0}= \\
& \frac{3 \rho_{0} U^{5 / 3}\left(128 K T_{0}\right)^{1 / 3}(1.1774)^{2 / 3}}{4\left(3 \pi e B_{0}\right)^{1 / 3}(\Delta S)^{2 / 3}} \boldsymbol{e}_{x} .
\end{aligned}
$$

The variable we want to solve in Eq. (46) is $\Delta S$. All other variables in the above equation can be obtained from the background medium models and the observed BBF data. The $\Delta S$ and $r$ are what we want to know about the equatorial cross section of a flow burst. From Eqs. (40), (41), and (44), the cross-tail width of flow burst $\Delta Y$ is given by

$$
\Delta Y=\left(\frac{128 K T_{0}(1.1774)^{2} \Delta S}{3 \pi e B_{0} U}\right)^{1 / 3}
$$

The value of $U$ is taken as $550 \mathrm{~km} \mathrm{~s}^{-1}$ from panel b of Fig. 6 of the work by Angelopoulos et al. (1992). The information about $\boldsymbol{B}$ and $\delta \boldsymbol{B}$ in the flow can be obtained indirectly from panel $d$ of the same figure. The ratio of the ion density in the downflow to that in the upflow is about 0.92 $\left(0.24 \mathrm{~cm}^{-3} / 0.26 \mathrm{~cm}^{-3}\right)$ from the panel. We would like to set this value as the ratio of plasma thermal pressure in a fully developed flow to that of the background medium. It is worth noting that this value is also close to the result of the fourth panel $(t=3)$ of Fig. 9 of the work by Chen and Wolf (1999). Substituting this ratio into Eq. (33), we would get $B$ and $\delta B$. Their directions are taken the same as a field line. This field line is taken from Tsyganenko (96) magnetospheric magnetic field model (Tsyganenko, 1995) (with the parameters set as: the solar wind density 6 per cubic centimeter, velocity $380 \mathrm{~km} \mathrm{~s}^{-1}$, IMF $B_{z}=5 \mathrm{nT}$ and Dst $=0 \mathrm{nT}$ ). This field line has equatorial plane crossing point $\mathrm{GSM} X=-10.0 R_{\mathrm{E}}$. The reason to choose this field line is simply to get a dipolar field line from an observation-based model. The shape of this field

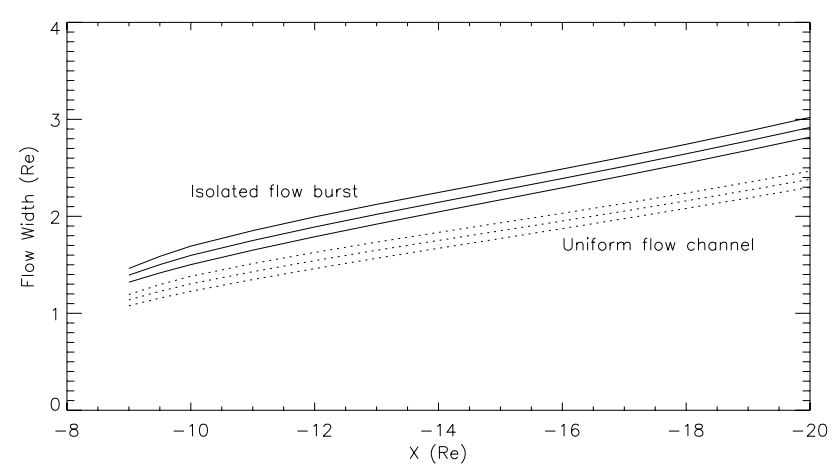

Fig. 7. Cross-tail width of fast flow. The three solid lines are for the isolated flow burst case (from top to bottom IMF $=-7.5 \mathrm{nT}$, $-5.0 \mathrm{nT},-2.5 \mathrm{nT})$; the three dashed lines are for uniform flow channel case (from top to bottom IMF $=-7.5 \mathrm{nT},-5.0 \mathrm{nT},-2.5 \mathrm{nT}$ ).

line makes the study of the effect of dipolarization of magnetic field in the fast flow possible.

Please note that the plasma parameters and magnetic field we use in this calculation are taken from Tsyganenko tail plasma sheet model (Tsyganenko and Mukai, 2003) and Tsyganenko (96) magnetospheric magnetic field model (Tsyganenko, 1995), but not from the unstable domain we constructed in Sect. 2. In these models, the solar wind parameters are chosen as density 6 per cubic centimeter, velocity $380 \mathrm{~km} \mathrm{~s}^{-1}$ and IMF $B_{z}=-2.5 \mathrm{nT}, B_{z}=-5 \mathrm{nT}$ and $B_{z}=$ $-7.5 \mathrm{nT}$, respectively. The Dst index is set equal to $-80 \mathrm{nT}$. Figure 6 gives a comparison of the dipolar field line with the stretched ones.

Figure 7 gives the cross-tail width of flow burst $\Delta Y$ as the function of down tail distance. It is important to remember that the plots in the present study can not be interpreted as the evolution of the variable as the flow burst moving earthward. Figure 8 gives the ratio of the $x$ dimension to the $y$ dimension of a FB. This ratio is consistent with the shape of the mushroom head some MHD simulations produced (Birn et al., 2004; Guzdar et al., 2010). Figure 9 gives the crosssection area $\Delta S$ of the mushroom head. 


\subsection{Width of uniform flow channel}

For the second case, a uniform flow channel, the velocitycaused pressure gradient term disappears. Thus Eq. (34) reduces to

$\frac{1}{\mu_{0}}(\boldsymbol{B} \cdot \nabla) \delta \boldsymbol{B}+\frac{1}{\mu_{0}}(\delta \boldsymbol{B} \cdot \nabla) \boldsymbol{B}_{0}+\mu \nabla^{2} \boldsymbol{u}=0$.

We now shift back to a satellite-based Cartesian coordinates with the same axial orientations as in the previous case. In this reference frame the Gaussian distribution of the velocity takes the form

$\boldsymbol{u}=U \exp \left(-\frac{y^{2}}{2 \sigma^{2}}\right) \boldsymbol{e}_{x}$

The flow induced drag force $\boldsymbol{F}_{\mathrm{d}}$ becomes

$\boldsymbol{F}_{\mathrm{d}}=-\frac{64 \rho_{0} U K T_{0}(1.1774)^{2}}{3 \pi e B_{0}(\Delta Y)^{2}} \boldsymbol{e}_{x}$

Substituting Eq. (50) into Eq. (48), we obtain

$\frac{1}{\mu_{0}}(\boldsymbol{B} \cdot \nabla) \delta \boldsymbol{B}+\frac{1}{\mu_{0}}(\delta \boldsymbol{B} \cdot \nabla) \boldsymbol{B}_{0}=\frac{64 \rho_{0} U K T_{0}(1.1774)^{2}}{3 \pi e B_{0}(\Delta Y)^{2}} \boldsymbol{e}_{x}$.

In this case, only the information about the flow channel width $\Delta Y$ would be abstracted. By plotting results in Fig. 7, we could see that in this case the flow channel width is smaller than the cross-tail width of the isolated flow burst. This is because in the uniform flow channel, viscosity is the only force that balances the magnetic buoyancy force, a narrower channel width would raise the drag force to the required value. While in the isolated flow burst case, both velocity-caused pressure gradient and viscosity (in present study the ratio of the former to the later happens to be $2: 1$ ) take part in the force balance, a wider width would be allowed.

A direct support of this result can be found from observations. For example, Sergeev et al. (1996) identified (Fig. 3 of their work) two BBFs events on 31 March 1979 by satellites ISEE 1 and 2. Event h1 took place at 15:55 UT and event h2 took place at 15:58 UT. These two events could be considered taking place in the same background environment. Event $\mathrm{h} 1$ lasted for two minutes and event h2 lasted about four minutes. Although event h2 did not last over ten minutes, it would be reasonable to think that it consists of more than two closely spaced BBFs. Based on the data of Table 1 in the same work, that is, the difference between the normals of discontinuity of BBFs fronts at two spacecraft and the spacing of two spacecraft $0.3 R_{\mathrm{E}}$, for a circular shape of the front boundary, event h1 would have a diameter of $2.46 R_{\mathrm{E}}$ (corresponding to 14 degree normal difference) and event h2 would have a diameter of $2.15 R_{\mathrm{E}}$ (corresponding to 16 degree normal difference). Our result is obtained under the assumption of the same speed $U$ at two extreme cases.

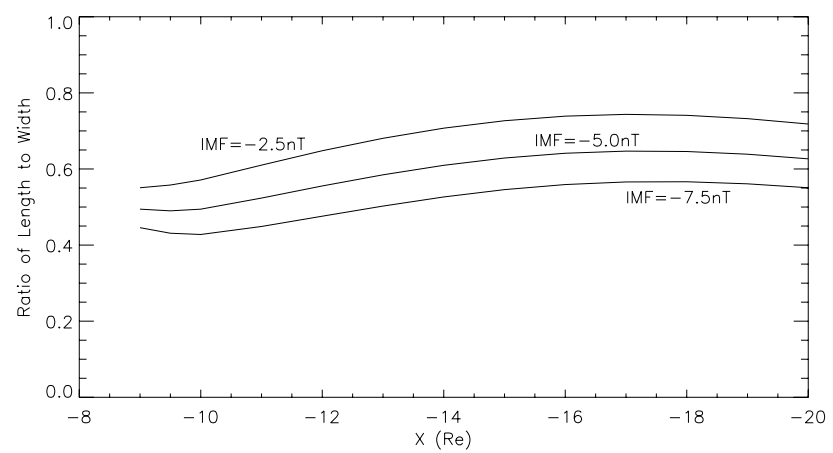

Fig. 8. Ratio of the $x$ dimension to the $y$ dimension of the crosssection area of fast flow.

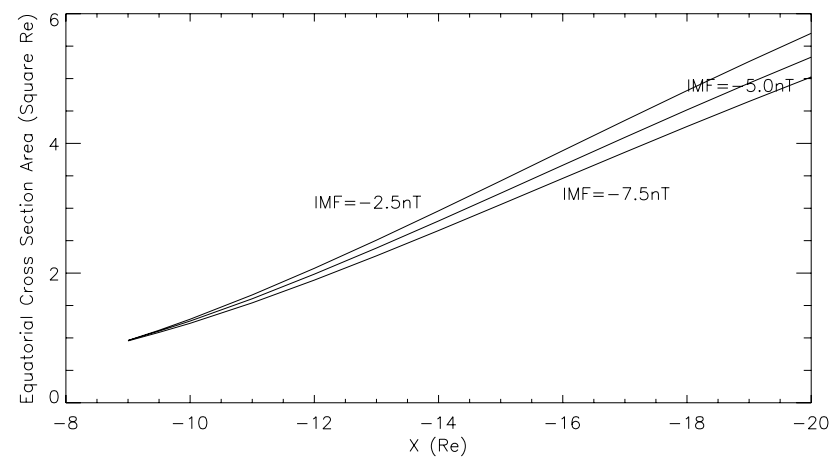

Fig. 9. Equatorial cross-section area of fast flow.

However, the lower speed $\left(\sim 200 \mathrm{~km} \mathrm{~s}^{-1}\right)$ of event $\mathrm{h} 1$ and the higher speed (over $400 \mathrm{~km} \mathrm{~s}^{-1}$ ) of event h2 strengthen the conclusion even more, because for a later extreme case (i.e., the uniform flow channel), the cross-tail width is an increasing function of the speed of BBFs. The reason behind this is that with the speed increase, the velocity gradient increases and thus the friction force. In order to balance the other forces, a wider width is required to lower the velocity gradient.

The real flow pattern in BBFs is somewhere between these two extreme cases. We could readily draw from Fig. 7 the conclusion that the cross-tail width of fast flow is in the range of 1 to $3 R_{\mathrm{E}}$ in the region between 9 and $20 R_{\mathrm{E}}$ down tail of the earth. These results are in agreement with the observations of BBFs on the central plasma sheet (Angelopoulos et al., 1996; Sergeev et al., 1996; Nakamura et al., 2004).

\section{Discussion and summary}

Before the discovery of BBFs in the plasma sheet, the flow velocity there is considered low, and viscosity did not get much attention. With the observation of fast flows, our view of viscosity needs some adjustment. The two flanks of fast flow are indeed two layers of large gradient of velocity in dawn-dusk direction. The range of the observed width of 
BBFs in the cross-tail direction ( 1 to $3 R_{\mathrm{E}}$ ) may be treated as the evidence of the existence of viscosity. By substituting the cross-tail width of BBFs in the denominator of the diffusion term of momentum equation, a friction force can be produced, which is inverse proportional to the square of width of BBFs, its range would cover almost one order of magnitude with $1-3 R_{\mathrm{E}}$ range of cross-tail width. Since the observations of BBFs are carried out in the region 10 to $20 R_{\mathrm{E}}$ down the tail, not covering a very large part of the magnetosphere, friction force in this range is capable to hold its position in the momentum equation under different states of the magnetosphere.

Any MHD theory that does not include the diffusion term in the momentum equation would have difficulty in finding the determinants of the cross-tail width of the fast flow just inside the unstable domain. Secondary derivative of coordinate $y$ in the dawn-dusk direction is mathematically crucial to this $y$ dimension problem. The exclusion of the diffusion term in momentum equation (Guzdar et al., 2010) led to an "outside source" explanation of the cross-tail width by seeding. The inclusion of the diffusion term may be an explanation why Lapenta and Bettarini (2011) can find a solution "inside".

In Sect. 2, we impose the condition of incompressibility of plasma. Such an assumption seems valid since the distance an MHD wave could travel in the time of the inverse of growth rate is well beyond the unstable domain extension $(d)$, convection cells would have enough time to set up. On the other hand, the real compressible medium may be more unstable, it would probably has smaller half wave length $(\pi / k)$ of the most unstable mode than the incompressible one because the unstable mode does not have to deal with the overall friction of the whole convection cell at the beginning moment.

Our results suggest that supposing the unstable domain is produced by magnetic reconnection, then the width of $x$ line, the half wave length $(\pi / k)$ of the most unstable mode and the width $(\Delta Y)$ of BBFs may be different. The latter two are closely related due to the nonlinear evolution of flow. The relation of the width of $x$ line with the other two widths, however, is not clear.

The information as to how a flow burst separates itself from the rest of its initial neighbor is not available from our present study. We can only imagine that in the time of the inverse of growth rate, a flow burst forms and makes its own way earthward. This scenario thus gives us an estimation of the duration of fast flow as the inverse of growth rate, which turns out to be consistent with the duration of the FB. The relationship between the growth rate and the thickness of the plasma sheet in Sect. 2 is in agreement with the statistical analysis of Ma et al. (2009). They found the duration for the recovery phase flow is typically $48 \mathrm{~s}$ and those for the growth and expansion phases are $99 \mathrm{~s}$ and $103 \mathrm{~s}$, respectively. Figure 5 shows that the thickness of the plasma sheet in the recovery phase is about $2 R_{\mathrm{E}}$ and in the growth and expansion phases is about $0.8 R_{\mathrm{E}}$.

From the inviscid case considered in the appendix, we know that growth rate reaches its maximum when wave number is infinity. In such situation, growth rate is determined by the driver, the magnetic buoyancy force (in our approximation the magnetic field gradient in a curved magnetic field), and the inertia of the convection cell. However, when viscosity is involved in the process, situations change dramatically. The growth rate reaches its maximum when the overall viscosity caused drag on the convection cell reaches a minimum. In a given dimension $(d)$ domain, larger wave numbers will have a larger $x$ component of the friction, however, because of the no slip boundary condition, the smaller wave number will have a larger $y$ component of the friction. Consequently, there is a compromise between the $x$ and $y$ components of the friction, the most unstable mode takes place with finite wave number.

With the increased depletion of the bubble (larger number of SP in Figs. 2 and 3), its size decreases and it becomes less stable. The positive correlation between the dimension of domain and the width of the bubble suggests that this result is consistent with the simulation by Birn et al. (2004) which showed that speed of flow is inversely correlated with the width of bubble.

The factors that have significant influence on the most unstable mode are viscosity, the dimension of the unstable domain, the thickness of the plasma sheet and gradient of the magnetic field.

In summary, we study two snap shots of the life of fast flow, one at its onset, the other when it is fully developed. Interchange instability analysis of domains with positive tailward gradient of magnetic field yields the most unstable mode with its half wave length comparable to the cross-tail width of a flow burst. The thickness of the plasma sheet $(0.8$ to $2 R_{\mathrm{E}}$ ) for the most unstable mode is also comparable to the width of BBFs in the north-south direction (1.5 to $2 R_{\mathrm{E}}$ ). The ion Larmor radius plays an important role as half effective mean free path length in earth's magnetosphere. Using empirical magnetic field and plasma models for earth's plasma sheet, the force balance analysis of fast flow gives the range of its cross-section area as one to several square $R_{\mathrm{E}}$ and cross-tail width as 1 to $3 R_{\mathrm{E}}$ which turns out to be in agreement with the observations.

\section{Appendix A}

\section{The growth rate of the most unstable mode}

Eliminating $\delta p$ from Eqs. (14) and (18) and substituting $\delta B$ from Eq. (13) leads to

$$
\begin{gathered}
D\left[-\frac{1}{k^{2}} \rho n D u_{x}+\frac{1}{k^{2}} \mu\left(D^{2}-k^{2}\right) D u_{x}+\frac{1}{k^{2}}(D \mu)\left(D^{2}+k^{2}\right) u_{x}\right] \\
=-\rho n u_{x}+\frac{2 B_{0} D B_{0}}{n \mu_{0} R} u_{x}+\mu\left(D^{2}-k^{2}\right) u_{x}+2(D \mu)\left(D u_{x}\right) .
\end{gathered}
$$


This is the equation satisfied by $u_{x}$ in differential form.

Substituting Eq. (13) into Eqs. (14) and (18), we have

$$
\begin{aligned}
& D \delta p=-\rho n u_{x}+\frac{1}{n \mu_{0}}\left(D B_{0}\right)^{2} u_{x}+\frac{B_{0}}{n \mu_{0}} D\left(u_{x} D B_{0}\right) \\
& +\frac{1}{n \mu_{0} R}\left(D B_{0}^{2}\right) u_{x}-\mu k^{2} u_{x}+\mu D^{2} u_{x}+2(D \mu)\left(D u_{x}\right), \\
& k^{2} \delta p=-\rho n D u_{x}-k^{2} \mu D u_{x}+k^{2} u_{x}(D \mu) \\
& \quad+D\left(\mu D^{2} u_{x}\right)+k^{2} \frac{D B_{0}^{2}}{2 n \mu_{0}} u_{x} .
\end{aligned}
$$

Suppose we have two solutions subscripted by $i$ and $j$. Multiplying Eq. (A2) satisfied by solution $i$ with $u_{j x}$ and integrating over the range of $x$, we get

$$
\begin{aligned}
\int_{-d}^{d} u_{j x} D \delta p_{i} \mathrm{~d} x & =-\int_{-d}^{d} \rho n_{i} u_{i x} u_{j x} \mathrm{~d} x \\
& +\int_{-d}^{d} \frac{1}{n_{i} \mu_{0}}\left(D B_{0}\right)^{2} u_{i x} u_{j x} \mathrm{~d} x \\
& +\int_{-d}^{d} \frac{B_{0}}{n_{i} \mu_{0}} u_{j x} D\left(u_{i x} D B_{0}\right) \mathrm{d} x \\
& +\int_{-d}^{d} \frac{1}{n_{i} \mu_{0} R}\left(D B_{0}^{2}\right) u_{i x} u_{j x} \mathrm{~d} x \\
& -\int_{-d}^{d} \mu k^{2} u_{i x} u_{j x} \mathrm{~d} x+\int_{-d}^{d} u_{j x} \mu D^{2} u_{i x} \mathrm{~d} x \\
& +\int_{-d}^{d} 2 u_{j x}(D \mu)\left(D u_{i x}\right) \mathrm{d} x .
\end{aligned}
$$

Multiplying Eq. (A3) satisfied by solution $i$ with $D u_{j x}$ and integrating over the range of $x$, one gets

$$
\begin{aligned}
-\int_{-d}^{d} \delta p_{i} D u_{j x} \mathrm{~d} x & =\int_{-d}^{d} \frac{n_{i}}{k^{2}} \rho\left(D u_{i x}\right)\left(D u_{j x}\right) \mathrm{d} x \\
& +\int_{-d}^{d} \mu\left(D u_{i x}\right)\left(D u_{j x}\right) \mathrm{d} x \\
& -\int_{-d}^{d} u_{i x}(D \mu) D u_{j x} \mathrm{~d} x \\
& -\int_{-d}^{d} \frac{1}{k^{2}}\left(D u_{j x}\right) D\left(\mu D^{2} u_{i x}\right) \mathrm{d} x \\
& -\int_{-d}^{d} \frac{D B_{0}^{2}}{2 n_{i} \mu_{0}} u_{i x} D u_{j x} \mathrm{~d} x .
\end{aligned}
$$

Using boundary condition (Eq. 19), integrating by parts the left side term and the fourth term on the right side of Eq. (A5), and then combining with Eq. (A4), we obtain

$$
\begin{aligned}
& \int_{-d}^{d}\left(\frac{n_{i}}{k^{2}} \rho+\mu\right)\left(D u_{i x}\right)\left(D u_{j x}\right) \mathrm{d} x \\
& +\frac{1}{k^{2}} \int_{-d}^{d} \mu\left(D^{2} u_{i x}\right)\left(D^{2} u_{j x}\right) \mathrm{d} x-\int_{-d}^{d}(D \mu) u_{i x} D u_{j x} \mathrm{~d} x \\
& -\int_{-d}^{d} \frac{D B_{0}^{2}}{2 n_{i} \mu_{0}} u_{i x} D u_{j x} \mathrm{~d} x=-\int_{-d}^{d} \rho n_{i} u_{i x} u_{j x} \mathrm{~d} x \\
& +\int_{-d}^{d} \frac{1}{n_{i} \mu_{0}}\left(D B_{0}\right)^{2} u_{i x} u_{j x} \mathrm{~d} x+\int_{-d}^{d} \frac{B_{0}}{n_{i} \mu_{0}} u_{j x} D\left(u_{i x} D B_{0}\right) \mathrm{d} x \\
& +\int_{-d}^{d} \frac{1}{n_{i} \mu_{0} R}\left(D B_{0}^{2}\right) u_{i x} u_{j x} \mathrm{~d} x-\int_{-d}^{d} \mu k^{2} u_{i x} u_{j x} \mathrm{~d} x \\
& +\int_{-d}^{d} u_{j x} \mu D^{2} u_{i x} \mathrm{~d} x+\int_{-d}^{d} 2 u_{j x}(D \mu)\left(D u_{i x}\right) \mathrm{d} x .
\end{aligned}
$$

Integrating by parts the third term on the right side of Eq. (A6) and rearranging the equation, we get

$$
\begin{aligned}
& -n_{i} \int_{-d}^{d} \rho\left[u_{i x} u_{j x}+\frac{1}{k^{2}}\left(D u_{i x}\right)\left(D u_{j x}\right)\right] \mathrm{d} x \\
& +\int_{-d}^{d} \frac{1}{n_{i} \mu_{0}}\left(D B_{0}\right)^{2} u_{i x} u_{j x} \mathrm{~d} x \\
& -\int_{-d}^{d} \frac{1}{n_{i} \mu_{0}} u_{i x}\left(D B_{0}\right) D\left(B_{0} u_{j x}\right) \mathrm{d} x \\
& +\int_{-d}^{d} \frac{1}{n_{i} \mu_{0} R}\left(D B_{0}^{2}\right) u_{i x} u_{j x} \mathrm{~d} x+\int_{-d}^{d} \frac{D B_{0}^{2}}{2 n_{i} \mu_{0}} u_{i x} D u_{j x} \mathrm{~d} x \\
& =\int_{-d}^{d} \mu\left[k^{2} u_{i x} u_{j x}+\left(D u_{i x}\right)\left(D u_{j x}\right)\right. \\
& \left.+\frac{1}{k^{2}}\left(D^{2} u_{i x}\right)\left(D^{2} u_{j x}\right)\right] \mathrm{d} x \\
& -\int_{-d}^{d} u_{j x}\left[\mu D^{2} u_{i x}+(D \mu)\left(D u_{i x}\right)\right] \mathrm{d} x
\end{aligned}
$$




$$
\begin{aligned}
& -\int_{-d}^{d} u_{j x}(D \mu)\left(D u_{i x}\right) \mathrm{d} x \\
& -\int_{-d}^{d} u_{i x}(D \mu) D u_{j x} \mathrm{~d} x .
\end{aligned}
$$

Expanding the third term on the left side and contracting the second, third and fourth terms on the right side of Eq. (A7), we obtain

$$
\begin{aligned}
& -n_{i} \int_{-d}^{d} \rho\left[u_{i x} u_{j x}+\frac{1}{k^{2}}\left(D u_{i x}\right)\left(D u_{j x}\right)\right] \mathrm{d} x \\
& +\int_{-d}^{d} \frac{1}{n_{i} \mu_{0} R}\left(D B_{0}^{2}\right) u_{i x} u_{j x} \mathrm{~d} x \\
& =\int_{-d}^{d} \mu\left[k^{2} u_{i x} u_{j x}+\left(D u_{i x}\right)\left(D u_{j x}\right)\right. \\
& \left.+\frac{1}{k^{2}}\left(D^{2} u_{i x}\right)\left(D^{2} u_{j x}\right)\right] \mathrm{d} x \\
& -\int_{-d}^{d} u_{j x} D\left[\mu\left(D u_{i x}\right)\right] \mathrm{d} x-\int_{-d}^{d}(D \mu) D\left(u_{i x} u_{j x}\right) \mathrm{d} x .
\end{aligned}
$$

Integrating by parts the second and third terms on the right side of Eq. (A8) leads to

$$
\begin{aligned}
& -n_{i} \int_{-d}^{d} \rho\left[u_{i x} u_{j x}+\frac{1}{k^{2}}\left(D u_{i x}\right)\left(D u_{j x}\right)\right] \mathrm{d} x \\
& +\int_{-d}^{d} \frac{1}{n_{i} \mu_{0} R}\left(D B_{0}^{2}\right) u_{i x} u_{j x} \mathrm{~d} x=\int_{-d}^{d}\left(D^{2} \mu\right) u_{i x} u_{j x} \mathrm{~d} x \\
& +\int_{-d}^{d} \mu\left[k^{2} u_{i x} u_{j x}+2\left(D u_{i x}\right)\left(D u_{j x}\right)\right. \\
& \left.+\frac{1}{k^{2}}\left(D^{2} u_{i x}\right)\left(D^{2} u_{j x}\right)\right] \mathrm{d} x .
\end{aligned}
$$

Subtracting the above equation with the result of exchanging $i$ and $j$ in it, we have

$$
\begin{array}{r}
\left(n_{j}-n_{i}\right)\left\{\int_{-d}^{d} \rho\left[u_{i x} u_{j x}+\frac{1}{k^{2}}\left(D u_{i x}\right)\left(D u_{j x}\right)\right] \mathrm{d} x\right. \\
\left.+\frac{1}{n_{i} n_{j}} \int_{-d}^{d} \frac{1}{\mu_{0} R}\left(D B_{0}^{2}\right) u_{i x} u_{j x} \mathrm{~d} x\right\}=0,
\end{array}
$$

which means, for $n_{i} \neq n_{j}$,

$$
\begin{aligned}
& \int_{-d}^{d} \rho\left[u_{i x} u_{j x}+\frac{1}{k^{2}}\left(D u_{i x}\right)\left(D u_{j x}\right)\right] \mathrm{d} x \\
& +\frac{1}{n_{i} n_{j}} \int_{-d}^{d} \frac{1}{\mu_{0} R}\left(D B_{0}^{2}\right) u_{i x} u_{j x} \mathrm{~d} x=0 .
\end{aligned}
$$

If $n_{i}$ should be complex, we can suppose $n_{i}$ and $n_{j}$ are complex conjugates, then Eq. (A11) becomes

$$
\begin{aligned}
& \int_{-d}^{d} \rho\left[\left|u_{x}\right|^{2}+\frac{1}{k^{2}}\left|\left(D u_{x}\right)\right|^{2}\right] \mathrm{d} x \\
& +\frac{1}{|n|^{2}} \int_{-d}^{d} \frac{1}{\mu_{0} R}\left(D B_{0}^{2}\right)\left|u_{x}\right|^{2} \mathrm{~d} x=0 .
\end{aligned}
$$

The above equation cannot be true if $D B_{0}^{2}$ is everywhere positive so that in this case $n$ must be real.

\section{A1 The variational principle}

Letting $i=j$ in Eq. (A9) and dropping the subscripts, we obtain the equation satisfied by $u_{x}$ in integral form.

$$
\begin{aligned}
& n \int_{-d}^{d} \rho\left[u_{x}^{2}+\frac{1}{k^{2}}\left(D u_{x}\right)^{2}\right] \mathrm{d} x-\int_{-d}^{d} \frac{1}{n \mu_{0} R}\left(D B_{0}^{2}\right) u_{x}^{2} \mathrm{~d} x \\
& =-\int_{-d}^{d}\left\{\mu\left[k^{2} u_{x}^{2}+2\left(D u_{x}\right)^{2}+\frac{1}{k^{2}}\left(D^{2} u_{x}\right)^{2}\right]\right. \\
& \left.+\left(D^{2} \mu\right) u_{x}^{2}\right\} \mathrm{d} x .
\end{aligned}
$$

Defining three quantities as following,

$$
\begin{aligned}
I_{1} & =\int_{-d}^{d} \rho\left[u_{x}^{2}+\frac{1}{k^{2}}\left(D u_{x}\right)^{2}\right] \mathrm{d} x \\
I_{2} & =\int_{-d}^{d} \frac{1}{\mu_{0} R}\left(D B_{0}^{2}\right) u_{x}^{2} \mathrm{~d} x, \\
I_{3} & =\int_{-d}^{d}\left\{\mu\left[k^{2} u_{x}^{2}+2\left(D u_{x}\right)^{2}+\frac{1}{k^{2}}\left(D^{2} u_{x}\right)^{2}\right]\right. \\
& \left.+\left(D^{2} \mu\right) u_{x}^{2}\right\} \mathrm{d} x,
\end{aligned}
$$

and taking variation of Eq. (A13), we obtain

$$
-\left(I_{1}+\frac{1}{n^{2}} I_{2}\right) \delta n=n \delta I_{1}-\frac{1}{n} \delta I_{2}+\delta I_{3} .
$$


The variation of $I_{1}$ can be represented by

$\delta I_{1}=\int_{-d}^{d} \rho\left[2 u_{x} \delta u_{x}+\frac{1}{k^{2}} 2\left(D u_{x}\right) D \delta u_{x}\right] \mathrm{d} x$.

Integrating by part the second term on the right side of above equation, we get

$\delta I_{1}=2 \int_{-d}^{d} \delta u_{x}\left[\rho u_{x}-\frac{1}{k^{2}} D\left(\rho D u_{x}\right)\right] \mathrm{d} x$.

The variation of $I_{2}$ can be represented by

$$
\begin{array}{r}
\delta I_{2}=\int_{-d}^{d} \frac{1}{\mu_{0} R}\left(D B_{0}^{2}\right) 2 u_{x} \delta u_{x} \mathrm{~d} x= \\
\quad 2 \int_{-d}^{d} \delta u_{x} \frac{1}{\mu_{0} R}\left(D B_{0}^{2}\right) u_{x} \mathrm{~d} x
\end{array}
$$

The variation of $I_{3}$ can be represented by

$$
\begin{aligned}
\delta I_{3} & =\int_{-d}^{d}\left\{\mu \left[k^{2} 2 u_{x} \delta u_{x}+4\left(D u_{x}\right) D \delta u_{x}\right.\right. \\
& \left.\left.+\frac{1}{k^{2}} 2\left(D^{2} u_{x}\right) D^{2} \delta u_{x}\right]+\left(D^{2} \mu\right) 2 u_{x} \delta u_{x}\right\} \mathrm{d} x
\end{aligned}
$$

Integrating by parts the second term on the right side of above equation once and the third term on the same side twice, we get

$$
\begin{aligned}
\delta I_{3} & =2 \int_{-d}^{d} \delta u_{x}\left[k^{2} \mu u_{x}-2 D\left(\mu D u_{x}\right)\right. \\
& \left.+\left(D^{2} \mu\right) u_{x}+\frac{1}{k^{2}} D^{2}\left(\mu D^{2} u_{x}\right)\right] \mathrm{d} x
\end{aligned}
$$

Substituting Eqs. (A19), (A20) and (A22) into Eq. (A17) and rearranging, we have

$$
\begin{aligned}
& \frac{1}{2} k^{2}\left(I_{1}+\frac{1}{n^{2}} I_{2}\right) \frac{\delta n}{n} \\
& =\int_{-d}^{d} \delta u_{x} D\left[\rho D u_{x}-\frac{\mu}{n}\left(D^{2}-k^{2}\right) D u_{x}\right. \\
& \left.-\frac{1}{n}(D \mu)\left(D^{2}+k^{2}\right) u_{x}\right] \mathrm{d} x \\
& -k^{2} \int_{-d}^{d} \delta u_{x}\left[\rho u_{x}-\frac{\mu}{n}\left(D^{2}-k^{2}\right) u_{x}-\frac{1}{n^{2}} \frac{1}{\mu_{0} R}\left(D B_{0}^{2}\right) u_{x}\right. \\
& \left.-\frac{2}{n}(D \mu) D u_{x}\right] \mathrm{d} x .
\end{aligned}
$$

The condition that for arbitrary $\delta u_{x}, \delta n$ is zero requires the sum of the integrands on the right side of above equation is zero, which is exactly Eq. (A1).

\section{A2 Inviscid case}

For inviscid case, Eq. (A13) reduces to

$$
\begin{aligned}
& n \int_{-d}^{d} \rho\left[u_{x}^{2}+\frac{1}{k^{2}}\left(D u_{x}\right)^{2}\right] \mathrm{d} x \\
& -\int_{-d}^{d} \frac{1}{n \mu_{0} R}\left(D B_{0}^{2}\right) u_{x}^{2} \mathrm{~d} x=0 .
\end{aligned}
$$

The solution for $n^{2}$ is

$n^{2}=\frac{\int\left(\frac{D B_{0}^{2}}{\mu_{0} R}\right) u_{x}^{2} \mathrm{~d} x}{\frac{1}{k^{2}} \int \rho\left(D u_{x}\right)^{2} \mathrm{~d} x+\int \rho u_{x}^{2} \mathrm{~d} x}$.

In a domain in which $D B_{0}^{2}$ is positive everywhere, $n$ would reaches its maximum value when $k$ approaches infinity.

Acknowledgements. I would like to thank Dick Wolf for helpful suggestions and comments on this work. This work was supported by National Nature Science Foundation of China, under grants (NSFC41074118, NSFC41374179, NSFC41121003) and Chinese Academy of Science key item KZCX2-EW-QN501 and KZZDEW-01-4.

Topical Editor C. Owen thanks two anonymous referees for their help in evaluating this paper.

\section{References}

Angelopoulos, V., Baumjohann, W., Kennel, C. F., Coroniti, F. V., Kivelson, M. G., Pellat, R., Walker, R. J., Lühr, H., and Paschmann, G.: Bursty bulk flows in the inner central plasma sheet, J. Geophys. Res., 97, 4027-4039, 1992.

Angelopoulos, V., Kennel, C. F., Coroniti, F. V., Pellat, R., Kivelson, M. G., Walker, R. J., Russell, C. T., Baumjohann, W., Feldman, W. C., and Gosling, J. T.: Statistical characteristics of bursty bulk flow events, J. Geophys. Res., 99, 21257-21280, 1994.

Angelopoulos, V., Coroniti, F. V., Kennel, C. F., Kivelson, M. G., Walker, R. J., Russell, C. T., McPherron, R. L., Sanchez, E., Meng, C.-I., Baumjohann, W., Reeves, G. D., Belian, R. D., Sato, N., Friis-Christensen, E., Sutcliffe, P. R., Yumoto, K., and Harris, T.: Multipoint analysis of a bursty bulk flow event on April 11, 1985, J. Geophys. Res., 101, 4967-4989, 1996.

Antonova, E. E. and Ovchinnikov, I. L.: Magnetostatically equilibrated plasma sheet with developed medium scale turbulence: Structure and implications for substorm dynamics, J. Geophys. Res., 104, 17289-17297, 1999.

Ashour-Abdalla, M., El-Alaoui, M., Goldstein, M. L., Zhou, M., Schriver, D., Richard, R., Walker, R., Kivelson, M. G., and 
Hwang, K.-J.: Observations and simulations of non-local acceleration of electrons in magnetotail magnetic reconnection events, Nature Phys., 7, 360-365, doi:10.1038/NPHYS1903, 2010.

Baumjohann, W., Paschmann, G., and Lühr, H.: Characteristics of high-speed ion flows in the plasma sheet, J. Geophys. Res., 95, 3801-3109, 1990.

Birn, J., Raeder, J., Wang, Y. L., Wolf, R. A., and Hesse, M.: On the propagation of bubbles in the geomagnetic tail, Ann. Geophys., 22, 1773-1786, doi:10.5194/angeo-22-1773-2004, 2004.

Borovsky, J. E., Elphic, R. C., Funsten, H. O., and Thomsen, M. F.: The Earth's plasma sheet as a laboratory for flow turbulence in high- $\beta$ MHD, J. Plasma Phys., 57, 1-34, 1997.

Chandrasekhar, S.: Hydrodynamic and Hydromagnetic stability, Claredon Press, Oxford, 1961.

Chen, C. X. and Wolf, R. A.: Interpretation of high-speed flows in the plasma sheet, J. Geophys. Res., 98, 21409-21419, 1993.

Chen, C. X. and Wolf, R. A.: Theory of thin-filament motion in Earth's magnetotail and its application to bursty bulk flows, J. Geophys. Res., 104, 14613-14626, 1999.

Eastwood, J. P., Sibeck, D. G., Slavin, J. A., Goldstein, M. L., Lavraud, B., Sitnov, M., Imber, S., Balogh, A., Lucek, E. A., and Dandouras, I.: Observations of multiple X-line structure in the Earth's magnetotail current sheet: A Cluster case study, Geophys. Res. Lett., 32, L11105, doi:10.1029/2005GL022509, 2005.

Ge, Y. S., Raeder, J., Angelopoulos, V., Gilson, M. L., and Runov, A.: Interaction of dipolarization fronts within multiple bursty bulk flows in global MHD simulations of a substorm on 27 February 2009, J. Geophys. Res., 116, A00123, doi:10.1029/2010JA015758, 2011.

George, S.: Physics of high temperature plasmas, Academic Press, New York, 1979.

Guzdar, P. N., Hassam, A. B., Swisdak, M., and Sitnov, M. I.: A simple MHD model for the formation of multiple dipolarization fronts, Geophys. Res. Lett., 37, L20102, doi:10.1029/2010GL045017, 2010.

Kauristie, K., Sergeev, V. A., Kubyshkina, M., Pulkkinen, T. I., Angelopoulos, V., Phan, T., Lin, R. P., and Slavin, J. A.: Ionospheric current signatures of transient plasma sheet, J. Geophys. Res., 105, 10677-10690, 2000.

Lapenta, G. and Bettarini, L.: Self-consistent seeding of the interchange instability in dipolarization fronts, Geophys. Res. Lett., 38, L11102, doi:10.1029/2011GL047742, 2011.

Ma, Y. D., Cao, J. B., Nakamura, R., Zhang, T. L., Reme, H., Dandouras, I., Lucek, E., and Dunlop, M.: Statistical analysis of earthward flow bursts in the inner plasma sheet during substorms, J. Geophys. Res., 114, A07215, doi:10.1029/2009JA014275, 2009.

Nakamura, M. S., Matsumoto, H., and Fujimoto, M.: Interchange instability at the leading part of reconnection jets, Geophys. Res. Lett., 29, 1247, doi:10.1029/2001GL013780, 2002.

Nakamura, R., Baumjohann, W., Mouikis, C., Kistler, L. M., Runov, A., Volwerk, M., Asano, Y., Voros, Z., Zhang, T. L., Klecker, B., Reme, H., and Balogh, A.: Spatial scale of high-speed flows in the plasma sheet observed by Cluster, Geophys. Res. Lett., 31, L09804, doi:10.1029/2004GL019558, 2004.

Ohtani, S., Shay, M. A., and Mukai, T.: Temporal structure of the fast convective flow in the plasma sheet: Comparison between observations and two-fluid simulations, J. Geophys. Res., 109, A03210, doi:10.1029/2003JA010002, 2004.
Panov, E. V., Nakamura, R., Baumjohann, W., Angelopoulos, V., Petrukovich, A. A., Retinò, A., Volwerk, M., Takada, T., Glassmeier, K.-H., McFadden, J. P., and Larson, D.: Multiple overshoot and rebound of a bursty bulk flow, Geophys. Res. Lett., 37 , L08103, doi:10.1029/2009GL041971, 2010.

Pontius Jr., D. H. and Wolf, R. A.: Transient flux tubes in the terrestrial magnetosphere, Geophys. Res. Lett., 17, 49-52, 1990.

Pritchett, P. L. and Coroniti, F. V.: Interchange and kink modes in the near-Earth plasma sheet and their associated plasma flows, Geophys. Res. Lett., 24, 2925-2928, 1997.

Pritchett, P. L. and Coroniti, F. V.: A kinetic ballooning/interchange instability in the magnetotail, J. Geophys. Res., 115, A06301, doi:10.1029/2009JA014752, 2010.

Pritchett, P. L. and Coroniti, F. V.: Plasma sheet disruption by interchange-generated flow intrusions, Geophys. Res. Lett., 38, L10102, doi:10.1029/2011GL047527, 2011.

Pritchett, P. L. and Coroniti, F. V.: Structure and consequences of the kinetic ballooning/interchange instability in the magnetotail, J. Geophys. Res., 118, 146-159, doi:10.1029/2012JA018143, 2013.

Raeder, J., Zhu, P., Ge, Y., and Siscoe, G.: Open Geospace General Circulation Model simulation of a substorm: Axial tail instability and ballooning mode preceding substrom onset, J. Geophys Res., 115, A00116, doi:10.1029/2010JA015876, 2010.

Runov, A., Angelopoulos, V., Sitnov, M. I., Sergeev, V. A., Bonnell, J., McFadden, J. P., Larson, D., Glassmeier, K.H., and Auster, U.: THEMIS observations of an earthwardpropagating dipolarization front, Geophys. Res. Lett., 36, L14106, doi:10.1029/2009GL038980, 2009.

Schindler, K.: A self-consistent theory of the tail of the magnetosphere, in Earth's magnetospheric processes, edited by: McCormac, B. M., 200 p., D. Reidel, Norwell, Mass, 1972.

Sergeev, V. A., Angelopoulos, V., Gosling, J. T., Cattell, C. A., and Russell, C. T.: Detection of localized, plasma-depleted flux tubes or bubbles in the midtail plasma sheet, J. Geophys. Res., 101, 10817-10826, 1996.

Sergeev, V. A., Liou, K., Meng, C. I., Newell, P. T., Brittnacher, M., Parks, G., and Reeves, G. D.: Development of auroral streamers in association with localized impulsive injections to the inner magnetotail, Geophys. Res. Lett., 26, 417-420, 1999.

Sergeev, V. A., Sauvaud, J. A., Popescu, D., Kovrazhkin, R. A., Liou, K., Newell, P. T., Brittnacher, M., Parks, G., Nakamura, R., Mukai, T., and Reeves, G. D.: Multiple-spacecraft observation of a narrow transient plasma jet in the Earth's plasma sheet, Geophys. Res. Lett., 27, 851-854, 2000.

Sergeev, V. A., Angelopoulos, V., Apatenkov, S., Bonnell, J., Ergun, R., Nakamura, R., McFadden, J. P., Larson, D., and Runov, A.: Kinetic structure of the sharp injection/depolarization front in the flow-braking region, Geophys. Res. Lett., 36, L21105, doi:10.1029/2009GL040658, 2009.

Sitnov, M. I. and Swisdak, M.: Onset of collisionless magnetic reconnection in two-dimensional current sheets and formation of dipolarization fronts, J. Geophys. Res., 116, A12216, doi:10.1029/2011JA016920, 2011.

Tsyganenko, N. A.: Modeling the Earth's magnetospheric magnetic-field confined within magnetopause, J. Geophys. Res., 100, 5599-5612, 1995. 
Tsyganenko, N. A. and Mukai, T.: Tail plasma sheet models derived from Geotail particle data, J. Geophys. Res., 108, 1136, doi:10.1029/2002JA009707, 2003.

Wiltberger, M., Pulkkinen, T. I., Lyon, J. G., and Goodrich, C. C.: MHD simulation of the magnetotail during the December 10, 1996, substorm, J. Geophys. Res., 105, 27649-27663, 2000.

Wolf, R. A., Wan, Y., Xing, X., Zhang, J.-C., and Sazykin, S.: Entropy and plasma sheet transport, J. Geophys. Res., 114, A00D05, doi:10.1029/2009JA014044, 2009.
Zhou, M., Ashour-Abdalla, M., Deng, X. H., Schriver, D., ElAlaoui, M., and Pang, Y.: THEMIS observation of multiple depolarization fronts and associated wave characteristics in the near-Earth magnetotail, Geophys. Res. Lett., 36, L20107, doi:10.1029/2009GL040663, 2009. 